\title{
Numerically satisfactory solutions of Kummer recurrence relations
}

\author{
Javier Segura • Nico M. Temme
}

Received: 7 February 2008 / Revised: 10 July 2008 / Published online: 9 September 2008

(C) Springer-Verlag 2008

\begin{abstract}
Pairs of numerically satisfactory solutions as $n \rightarrow \infty$ for the three-term recurrence relations satisfied by the families of functions ${ }_{1} \mathrm{~F}_{1}\left(a+\epsilon_{1} n ; b+\epsilon_{2} n ; z\right)$, $\epsilon_{i} \in \mathbb{Z}$, are given. It is proved that minimal solutions always exist, except when $\epsilon_{2}=0$ and $z$ is in the positive or negative real axis, and that ${ }_{1} \mathrm{~F}_{1}\left(a+\epsilon_{1} n ; b+\epsilon_{2} n ; z\right)$ is minimal as $n \rightarrow+\infty$ whenever $\epsilon_{2}>0$. The minimal solution is identified for any recurrence direction, that is, for any integer values of $\epsilon_{1}$ and $\epsilon_{2}$. When $\epsilon_{2} \neq 0$ the confluent $\operatorname{limit}_{\lim } \rightarrow \infty{ }_{1} \mathrm{~F}_{1}(\gamma b ; b ; z)=e^{\gamma z}$, with $\gamma \in \mathbb{C}$ fixed, is the main tool for identifying minimal solutions together with a connection formula; for $\epsilon_{2}=0$, $\lim _{a \rightarrow+\infty} \mathrm{F}_{1}(a ; b ; z) /{ }_{0} \mathrm{~F}_{1}(; b ; a z)=e^{z / 2}$ is the main tool to be considered.
\end{abstract}

\section{Mathematics Subject Classification (2000) $\quad 33 \mathrm{C} 15 \cdot 39 \mathrm{~A} 11 \cdot 41 \mathrm{~A} 60 \cdot$ 65D20}

\section{Introduction}

Linear three-term recurrence relations are useful tools for computing a larger number of special functions, and in particular, confluent (or Kummer) hypergeometric functions.

As is well known, the Kummer function ${ }_{1} \mathrm{~F}_{1}$, defined by

$$
{ }_{1} \mathrm{~F}_{1}(a ; b ; z)=\sum_{k=0}^{\infty} \frac{(a)_{n}}{(b)_{n}} \frac{z^{n}}{n !},
$$

\footnotetext{
J. Segura $(\bowtie)$

Departamento de Matemáticas, Estadística y Computación, Universidad de Cantabria, 39005 Santander, Spain

e-mail: javier.segura@unican.es

N. M. Temme

CWI, P.O. Box 94079, 1090 GB Amsterdam, The Netherlands

e-mail: nicot@cwi.nl
} 
satisfies linear relations with rational coefficients connecting any three functions with parameters differing by integer numbers.

In particular, given a family of Kummer functions

$$
y_{n}={ }_{1} \mathrm{~F}_{1}\left(a+\epsilon_{1} n ; b+\epsilon_{2} n ; z\right)
$$

for any pair $\left(\epsilon_{1}, \epsilon_{2}\right) \neq(0,0)$, with fixed values of $\epsilon_{i} \in \mathbb{Z}$, and with fixed complex values of $a, b$ and $z$, there exists a three-term recurrence relation

$$
y_{n+1}+\beta_{n} y_{n}+\alpha_{n} y_{n-1}=0,
$$

$\alpha_{n}$ and $\beta_{n}$ being rational functions of $a_{n}=a+\epsilon_{1} n, b_{n}=b+\epsilon_{2} n$ and $z$. Any of the recurrence relations, for any integer values of $\epsilon_{1}$ and $\epsilon_{2}$, can be obtained by suitably combining linear relations connecting contiguous confluent hypergeometric functions (see [1, Eqs. 13.4.1-13.4.6]). For example, if $\left(\epsilon_{1}, \epsilon_{2}\right)=(1,0)$ we have $\beta_{n}=\left(-2 a_{n}-z+b_{n}\right) / a_{n}, \alpha_{n}=1-b_{n} / a_{n} ;$ for $\left(\epsilon_{1}, \epsilon_{2}\right)=(0,1), \beta_{n}=\left(1-b_{n}-z\right) / z$, $\alpha_{n}=\left(b_{n}-1-a_{n}\right) / z$; and for the case $\left(\epsilon_{1}, \epsilon_{2}\right)=(1,1), \beta_{n}=\left(b_{n}-z-1\right) /\left(a_{n} z\right)$, $\alpha_{n}=-1 /\left(a_{n} z\right)$ (see [6, Chap. 4] for further details and examples). As a further explicit example, we will consider later the recurrences $\left(\epsilon_{1}, \epsilon_{2}\right)=( \pm 1, \pm 2)$.

A crucial point to be elucidated before using a linear three-term recurrence relation (TTRR) for computing a given function is the conditioning of the computation. In particular, when a recurrence admits a minimal (or recessive) solution $f_{n}$, that is, a solution such that

$$
\lim _{n \rightarrow+\infty} \frac{f_{n}}{g_{n}}=0
$$

for any other solution $g_{n}$ of the TTRR independent of $f_{n}$, this fact determines that only one of the two possible recurrence directions (increasing or decreasing $n$ ) is well conditioned, depending on the solution that is computed. Indeed, for computing the minimal solution, the forward recurrence (increasing $n$ ) is ill conditioned, because a small perturbation of the initial values (say $f_{0}$ and $f_{1}$ ) will introduce a component of a dominant solution, which will ultimately dominate for sufficiently large $n$. On the contrary, the forward numerical computation of a dominant solution $g_{n}$ with initial values $g_{0}$ and $g_{1}$, is well conditioned. Regarding computation in the backward direction (starting with large $n$ ), the evaluation of a minimal solution $f_{n}$ with decreasing $n$ is well conditioned; contrarily, a dominant solution should never be computed in the backward direction.

When a recurrence admits a minimal solution (unique except for a multiplicative factor), a pair of numerically satisfactory solutions should comprise the minimal solution and a dominant solution. From such a pair, any other solution can in principle be computed in a numerically stable way (although transitory effects are also possible [2]).

The case of Kummer recurrences contains, as particular cases, many important functions like Hermite and Laguerre polynomials and Bessel and Coulomb functions. However, a systematic analysis of the conditioning of Kummer recurrences in the complex plane was not considered until recently [3], when numerically satisfactory 
solutions were found for the Kummer recurrences satisfied by the functions (2) with $\left|\epsilon_{i}\right| \leq 1$. The main tools for identifying minimal and dominant solutions were Perron's theorem (see for instance, [6, Chap. 4]), together with uniform asymptotic estimates for Whittaker functions [4].

In the present paper, using asymptotic estimates for fixed complex $z$ we are able to find the minimal solution (when it exists) for any integer values of $\epsilon_{i}$, and a second independent solution. In other words, the problem of finding numerically satisfactory solutions for Kummer recurrences is completely solved, for all $\epsilon_{i} \in \mathbb{Z}$.

In the analysis, one has to consider two different asymptotic estimates: a wellknown estimate in terms of Bessel functions [8, p. 80] for the case $\epsilon_{2}=0$, and a new one for the cases $\epsilon_{2} \neq 0$. In the Appendix, we give details on obtaining the new asymptotic (and convergent) expansions corresponding to the case $\epsilon_{2} \neq 0$.

\section{Pairs of satisfactory solutions when $\epsilon_{2} \neq 0$}

The main two ingredients for finding the numerically satisfactory solutions are, first, an asymptotic estimate for large parameters and, second, a connection formula.

For $\epsilon_{2} \neq 0$ the asymptotic estimate is provided by the following limit relation:

$$
\lim _{b \rightarrow \infty}{ }_{1} \mathrm{~F}_{1}(\gamma b ; b ; z)=e^{\gamma z}
$$

$\gamma$ being a fixed value. This implies that, given two sequences $\left\{a_{n}\right\},\left\{b_{n}\right\}$ such that $a_{n} \rightarrow \infty$ and $b_{n} \rightarrow \infty$ with finite limit $\gamma=\lim _{n \rightarrow \infty} a_{n} / b_{n}$, we can write

$$
\lim _{n \rightarrow \infty}{ }_{1} \mathrm{~F}_{1}\left(a_{n} ; b_{n} ; z\right)=e^{\gamma z}
$$

One can understand (5) as a result of taking a double confluent limit as follows:

$$
\lim _{a, b \rightarrow \infty}{ }_{1} \mathrm{~F}_{1}(a ; b ; b z / a)={ }_{0} F_{0}(; ; z)=e^{z},
$$

with a fixed ratio $a / b$. Also, this limit reproduces the dominant behavior shown by the uniform asymptotic expansions of Olver [7, p. 261] and Dunster [4], considered in [3] for finding the condition of some Kummer recurrences. It is surprising, however, that such limit relation does not appear explicitly in the literature. In the Appendix, we provide additional details, including new asymptotic expansions of ${ }_{1} \mathrm{~F}_{1}(\gamma b ; b ; z)$ as $b$ becomes large, with fixed $\gamma \in \mathbb{C}$.

Regarding the analysis of the condition of the recurrences, the important point in (6) is that the resulting limit is a well-defined bounded function of $z$ and that, therefore

$$
\lim _{n \rightarrow+\infty} \frac{y_{n+1}^{(1)}(z)}{y_{n}^{(1)}(z)}=1
$$


for

$$
y_{n}^{(1)}(z)={ }_{1} \mathrm{~F}_{1}\left(a+\epsilon_{1} n ; b+\epsilon_{2} n ; z\right),
$$

when $\epsilon_{1}, \epsilon_{2} \neq 0$.

This relation also holds when $\epsilon_{1}=0$ : we can see this from the Maclaurin series (1) of the Kummer function, or by using

$$
{ }_{1} \mathrm{~F}_{1}(a ; b ; z)=e^{z} \mathrm{~F}_{1}(b-a ; b ;-z) .
$$

Therefore, except for $\epsilon_{2}=0,(8)$ always holds. Later, we consider separately the case $\epsilon_{2}=0$.

The second main ingredient is the well-known connection formula

$$
U(a, b, z)=\frac{\Gamma(1-b)}{\Gamma(a+1-b)}{ }_{1} \mathrm{~F}_{1}(a ; b ; z)+\frac{\Gamma(b-1)}{\Gamma(a)} z^{1-b}{ }_{1} \mathrm{~F}_{1}(a-b+1 ; 2-b ; z),
$$

which is already used in [3] for building accompanying solutions of the recurrence satisfied by $y_{n}^{(1)}(z)$ when $\left|\epsilon_{i}\right| \leq 1$. The $U$-function, when multiplied by appropriate $\Gamma$ factors, is a solution of the same recurrences as those for ${ }_{1} F_{1}$. This can be checked explicitly by verifying that the contiguous relations between ${ }_{1} \mathrm{~F}_{1}(a ; b ; z)$ and any two functions with contiguous parameters (that is, with one or two parameters differing in one unit) is the same; from these contiguous relations the rest of recurrences can be built. Because of this, also the second term in the right-hand side of (11) can be used for building solutions of the Kummer recurrences.

The $U$-function will not be crucial in the present analysis, because the two terms at the right-hand side of (11) allow the construction of numerically satisfactory pairs of solutions in all cases, except when $\epsilon_{2}=0$, in which case the $U$-function is minimal. We recall that a pair is said to be satisfactory when the minimal solution is included in the pair.

With this, we will show that the pair

$$
\begin{aligned}
\left\{y_{n}^{(1)}(z), y_{n}^{(2)}(z)\right\} & =\left\{{ }_{1} \mathrm{~F}_{1}\left(a_{n}, b_{n}, z\right), \lambda_{n} z^{-b_{n}}{ }_{1} \mathrm{~F}_{1}\left(a_{n}-b_{n}+1,2-b_{n}, z\right)\right\}, \\
\lambda_{n} & =\frac{\Gamma\left(b_{n}-1\right) \Gamma\left(a_{n}-b_{n}+1\right)}{\Gamma\left(1-b_{n}\right) \Gamma\left(a_{n}\right)}, \\
a_{n} & =a+\epsilon_{1} n, b_{n}=b+\epsilon_{2} n,
\end{aligned}
$$

is a satisfactory pair whenever $\epsilon_{2} \neq 0$.

As explained in $[3,5]$, it is convenient to use the reflection formula for the gamma function in order to avoid negative values of $n$. Then, it is assumed that the reflection formula

$$
\Gamma(\lambda-n)=\frac{(-1)^{n} \pi}{\sin (\pi \lambda) \Gamma(n+1-\lambda)}
$$


is used when $n$ appears with negative sign. The factors not depending on $n$ can, of course, be dropped.

Using (8), together with (12) and (13), it is a straightforward matter to check that, as $n \rightarrow+\infty$,

$$
\frac{y_{n+1}^{(1)}(z)}{y_{n}^{(1)}(z)} \sim 1, \frac{y_{n+1}^{(2)}(z)}{y_{n}^{(2)}(z)} \sim C\left(\begin{array}{c}
n \\
z
\end{array}\right)^{\epsilon_{2}} .
$$

When $\epsilon_{1}, \epsilon_{2} \neq 0$, the constant $C$ reads

$$
C=(-1)^{s}\left|\epsilon_{1}\right|^{-\epsilon_{1}}\left|\epsilon_{2}\right|^{2 \epsilon_{2}}\left|\epsilon_{1}-\epsilon_{2}\right|^{\epsilon_{1}-\epsilon_{2}}, \quad s=\frac{1}{2}\left(\operatorname{sign}\left(\epsilon_{1}\right)+\operatorname{sign}\left(\epsilon_{2}\right)\right)
$$

with $\operatorname{sign}(\epsilon)=+1$ when $\epsilon \geq 0$ and $\operatorname{sign}(\epsilon)=-1$ when $\epsilon<0$. When $\epsilon_{1}=0$ the same expression is valid by neglecting the factor $\left|\epsilon_{1}\right|^{-\epsilon_{1}}$.

The crucial information is the dependence on $n$ of the ratio $y_{n+1}^{(2)} / y_{n}^{(2)}$, showing that $y_{n}^{(2)}$ dominates over $y_{n}^{(1)}$ when $\epsilon_{2}>0$, which means that $y_{n}^{(1)}$ is minimal. The contrary happens when $\epsilon_{2}<0$.

This holds for any recurrence relation with $\epsilon_{2} \neq 0$ provided the solutions are defined (which, for instance, is not the case when $b \in \mathbb{Z}^{ \pm}$and $n \rightarrow \mp \infty$ ) and for all $z \in \mathbb{C}$; also, the argument breaks down for $z=0$, in which case the second solution is undefined or zero. In all these cases, the recurrence relation becomes singular in the sense that $\alpha_{n}$ becomes 0 or $\infty$ for some $n$ value. Therefore, the result holds when the recurrence is non-singular and can be applied in the corresponding recurrence direction. That is, the result is as general as can be.

It can be easily checked that all results corresponding to $\epsilon_{2} \neq 0$ described in [3] can be condensed in (7-10). For a further check, we consider the recurrences with $\epsilon_{1}= \pm 1$ and $\epsilon_{2}= \pm 2$ (abbreviated, the $( \pm 1, \pm 2$ ) recurrences), which have as particular cases Bessel and Coulomb wave functions; we will test the asymptotic estimates against the prediction of Perron's theorem [6, Theorem. 4.6]. In this case, the recurrence relation $y_{n+1}+\beta_{n} y_{n}+\alpha_{n} y_{n-1}=0$ satisfied by $y_{n}^{(1)}={ }_{1} \mathrm{~F}_{1}\left(a_{n} ; b_{n} ; z\right)$ has coefficients

$$
\begin{aligned}
& \beta_{n}=f_{n} c_{n}, c_{n}=\left[b_{n}\left(b_{n}-z-2\right)+2 z a_{n}\right] /\left(b_{n}-2\right), \\
& \alpha_{n}=-f_{n} b_{n}, \\
& f_{n}=\left(b_{n}^{2}-1\right) b_{n} /\left(a_{n}\left(b_{n}-a_{n}\right) z^{2}\right),
\end{aligned}
$$

when $a_{n}=a+n, b_{n}=b+2 n$ (which is the $(1,2)$ recurrence) and

$$
\beta_{n}=\frac{c_{n}}{b_{n}}, \quad \alpha_{n}=-\frac{1}{f_{n} b_{n}}
$$

when $a_{n}=a-n, b_{n}=b-2 n$ (the $(-1,-2)$ recurrence). 
Therefore, for the $(1,2)$ recurrence, we have

$$
\beta_{n} \sim \frac{16 n^{2}}{z^{2}}, \quad \alpha_{n} \sim-\frac{16 n^{2}}{z^{2}} .
$$

Perron's theorem guarantees that this recurrence relation admits a minimal solution. Further, it provides the asymptotic estimates for the minimal, $y_{n}^{(1)}$, and dominant solutions $y_{n}^{(2)}$ :

$$
\frac{y_{n}^{(1)}(z)}{y_{n-1}^{(1)}(z)} \sim-\frac{\alpha_{n}}{\beta_{n}} \sim 1, \quad \frac{y_{n}^{(2)}(z)}{y_{n-1}^{(2)}(z)} \sim-\frac{16 n^{2}}{z^{2}} .
$$

This prediction is, indeed, consistent with (14) and (15). ${ }_{1} \mathrm{~F}_{1}(a+n ; b+n ; z)$ is indeed minimal as $n \rightarrow+\infty$ for any set of complex parameters $a, b, z$. In particular, the regular Coulomb function $F_{L}$ is therefore minimal as $L \rightarrow \infty$ (and also, as a particular case, the Bessel function $J_{v}(z)$, as $\left.v \rightarrow+\infty\right)$.

For the $(-1,-2)$ recurrence, we have

$$
\beta_{n} \sim 1, \quad \alpha_{n} \sim-\frac{z^{2}}{16 n^{2}}
$$

and Perron's theorem is again positive with respect to the existence of minimal solution and provides the following asymptotic estimates for the minimal, $y_{n}^{(2)}$, and dominant solutions $y_{n}^{(1)}$ :

$$
\frac{y_{n}^{(1)}(z)}{y_{n-1}^{(1)}(z)} \sim 1, \quad \frac{y_{n}^{(2)}(z)}{y_{n-1}^{(2)}(z)} \sim-\beta_{n} \sim-\frac{z^{2}}{16 n^{2}} .
$$

We see that the minimal solution exists and that it is not $y_{n}^{(1)}(z)={ }_{1} \mathrm{~F}_{1}(a-n, b-2 n, z)$ in this case, but $y_{n}^{(2)}$.

\section{Satisfactory solutions when $\epsilon_{2}=0$}

This case was treated in [3], and we only mention this for completeness. It is worth observing that because of the Kummer relation (10), the functions ${ }_{1} \mathrm{~F}_{1}(b-a+$ $\left.\epsilon_{1} n ; b ;-z\right)$ and ${ }_{1} \mathrm{~F}_{1}\left(a-\epsilon_{1} n ; b ; z\right)$ satisfy the same recurrence relation, and that, hence, the recurrences $\left(\epsilon_{1}, 0\right)$ and $\left(-\epsilon_{1}, 0\right)$ are related.

Also, it is clear that the information for the case $(1,0)$ (and hence also $(-1,0)$ ) gives also all the information for other values $\epsilon_{1} \in \mathbb{Z}$, because the recurrence direction is the same.

For the case $(1,0)$ another type of confluent limit is considered, namely

$$
\lim _{a \rightarrow \infty} \mathrm{F}_{1}(a ; b ; z) /{ }_{0} \mathrm{~F}_{1}(; b ; a z)=e^{z / 2},
$$


which follows from the following relation between the ${ }_{0} \mathrm{~F}_{1}$ hypergeometric function and Bessel functions. We have

$$
{ }_{0} \mathrm{~F}_{1}(; b ; z)=\Gamma(b) z^{(1-b) / 2} I_{b-1}(2 \sqrt{z}),
$$

and the known asymptotic estimate (see $[8, \mathrm{p} .80])$

$$
{ }_{1} \mathrm{~F}_{1}(a ; b ; z) \sim e^{z / 2} \Gamma(b)(a z)^{(1-b) / 2} I_{b-1}(2 \sqrt{a z}), \quad a \rightarrow \infty .
$$

Similar estimations are available for the $U$-function. Considering (11), we see that when the $U$-function is multiplied by $\Gamma(a+1-b)$, the resulting function will satisfy the same recurrence (in the direction of varying $a$ ) as the function ${ }_{1} \mathrm{~F}_{1}(a ; b ; z)$. We have the known asymptotic behavior $[8$, p. 80]

$$
\Gamma(a+1-b) U(a, b, z) \sim 2(a z)^{(1-b) / 2} e^{z / 2} K_{b-1}(2 \sqrt{a z}) .
$$

With this, and using the fact that the Bessel function $I_{v}(w)\left(K_{v}(w)\right)$ is exponentially large (small) as $\Re w \rightarrow \infty$, it is clear that the pair

$$
\left\{y_{n}^{(1)}(z), y_{n}^{(2)}(z)\right\}=\left\{{ }_{1} \mathrm{~F}_{1}(a+n ; b ; z), \Gamma(a+1-b+n) U(a+n, b, z)\right\}
$$

is a numerically satisfactory pair and the $y_{n}^{(2)}$ is minimal except for $z$ at the negative real axis for the $(1,0)$ recurrence and at the positive real axis for the $(-1,0)$ recurrence. In these two latter cases, the pair of solutions is also satisfactory, but the functions become oscillatory as a function of the first parameter, as can be seen from (23) to (25), and no minimal solution exists.

Observe that this pair is also satisfactory when $\epsilon_{2}>0$, because we proved in that case that $y_{n}^{(1)}$ is minimal. We summarize this in the next section.

\section{Main results}

We summarize the main results of this paper in the following theorem. As mentioned before, it is assumed that the reflection formula (13) is applied when the gamma functions acquire a negative argument.

We use the notation

$$
a_{n}=a+\epsilon_{1} n, \quad b_{n}=b+\epsilon_{2} n, \quad n, \epsilon_{1}, \epsilon_{2} \in \mathbb{Z}, \quad a, b \in \mathbb{C} .
$$

Theorem 1 (Recurrences with $\epsilon_{2} \geq 0$ ) The pair of functions

$$
\left\{y_{n}^{(1)}, y_{n}^{(2)}\right\}=\left\{{ }_{1} F_{1}\left(a_{n} ; b_{n} ; z\right), \Gamma\left(1+a_{n}-b_{n}\right) U\left(a_{n}, b_{n}, z\right)\right\}
$$

is a numerically satisfactory pair of solutions of the $\left(\epsilon_{1}, \epsilon_{2}\right)$ three-term recurrence relation (3) when $\epsilon_{2}=0,1,2, \ldots$ 
The solution $y_{n}^{(1)}$ is minimal for all complex z as $n \rightarrow+\infty$ if $\epsilon_{2}>0$. When $\epsilon_{2}=0$, $y_{n}^{(2)}$ is minimal except when $\Im z=0$ and $\epsilon_{1} \Re z<0$, in which case no minimal solution exists.

Theorem 2 (Recurrences with $\epsilon_{2} \neq 0$ ) The pair of functions

$$
\begin{aligned}
& \left\{y_{n}^{(1)}(z), y_{n}^{(2)}(z)\right\}=\left\{{ }_{1} F_{1}\left(a_{n}, b_{n}, z\right), \lambda_{n} z^{-b_{n}}{ }_{1} F_{1}\left(a_{n}-b_{n}+1,2-b_{n}, z\right)\right\}, \\
& \lambda_{n}=\frac{\Gamma\left(b_{n}-1\right) \Gamma\left(a_{n}-b_{n}+1\right)}{\Gamma\left(1-b_{n}\right) \Gamma\left(a_{n}\right)},
\end{aligned}
$$

constitutes a pair of numerically satisfactory solutions whenever $\epsilon_{2} \neq 0$.

The solution $y_{n}^{(1)}$ is minimal when $\epsilon_{2}>0$ and $y_{n}^{(2)}$ is minimal when $\epsilon_{2}<0$. This holds for all complex values of $z$.

\section{Appendix: Two expansions for ${ }_{1} \mathrm{~F}_{1}(a ; b, z)$ as $b \rightarrow \infty$ with $a / b$ fixed}

A first glance at the limit (5) suggests that it can be understood from the power series definition of the Kummer function given in (1). If both $a$ and $b$ tend to infinity with $\gamma=a / b$ fixed, we observe, given

$$
\lim _{b \rightarrow \infty} \frac{(\gamma b)_{k}}{(b)_{k}} \rightarrow \gamma^{k}
$$

that then (5) holds term by term. A better way to confirm this result is by building asymptotic series as $b$ becomes large, as we are doing now in two different ways: by using the differential equation and by using integral representations.

An expansion obtained from the differential equation

The starting point is Kummer's differential equation

$$
z y^{\prime \prime}+(b-z) y^{\prime}-a y=0 .
$$

Now, because we expect that the limit (5) holds we write

$$
y=e^{\gamma z} w
$$

where $\gamma=a / b$, and then it is straightforward to verify that $w$ satisfies the following differential equation

$$
-z\left[w^{\prime \prime}+(2 \gamma-1) w^{\prime}+\gamma(\gamma-1) w\right]=b w^{\prime} .
$$


Now, we write the formal series

$$
w(z)=\sum_{k=0}^{\infty} A_{k}(z) b^{-k}
$$

with $A_{0}=1$, as corresponds with the fact that ${ }_{1} \mathrm{~F}_{1}(b ; b ; z)=\exp (z)$. Then equating equal powers of $b$ we find that the rest of the coefficients can be obtained recursively by means of the relation

$$
A_{k+1}(z)=-\int_{0}^{z} x\left[A_{k}^{\prime \prime}(x)+(2 \gamma-1) A_{k}^{\prime}(x)+\gamma(\gamma-1) A_{k}(x)\right] d x .
$$

For instance, the expansion with three terms reads:

$$
\begin{aligned}
& { }_{1} F_{1}(\gamma b ; b ; z) \sim E_{\gamma, b}(z)=e^{\gamma z}\left[1+\gamma(\gamma-1) \frac{z^{2}}{2 b}\left(-1+\frac{\phi(z)}{12 b}\right)+\mathcal{O}\left(b^{-3}\right)\right], \\
& \phi(z)=3 \gamma(\gamma-1) z^{2}+8(2 \gamma-1) z+12 .
\end{aligned}
$$

Clearly, for fixed $z$ and $\gamma$ the coefficients $A_{k}(z)$ are bounded. In addition, the expansion $E_{\gamma, b}(z)$ satisfies $E_{\gamma, b}(0)=1, E_{\gamma, b}^{\prime}(0)=\gamma=a / b$, as corresponds to the solution ${ }_{1} \mathrm{~F}_{1}$ of the differential equation.

In addition the derivatives at $z=0$ of the expansion up to order $\mathcal{O}\left(b^{-n}\right)$ at $z=0$ coincide up to $\mathcal{O}\left(b^{-n}\right)$ with the derivatives of ${ }_{1} F_{1}(\gamma b ; b ; z)$. This is not surprising, since we have applied the differential equation term by term in the asymptotic series.

Observe that the expansion is exact for $\gamma=0$ or $\gamma=1$, because all the coefficients except $A_{0}$ appear multiplied by the factor $\gamma(\gamma-1)$ and, obviously ${ }_{1} \mathrm{~F}_{1}(b ; b ; z)=e^{z}$ and ${ }_{1} \mathrm{~F}_{1}(0 ; b ; z)=1, b \neq 0$.

The above analysis can be supported by the proofs for more general cases given in [7, Chap. 10]. The expansion (34) holds for large complex $b$, uniformly with respect to bounded $z, \gamma=a / b \in \mathbb{C}$. No restriction has been considered when building the series. A more direct verification of the validity of such series, and therefore of the limit (5), is obtained by considering a related expansion obtained from integral representations. We recall that the limit (5) when $\gamma \in \mathbb{R}$ is the only ingredient used in analyzing the conditioning of the recurrences.

An expansion obtained from integrals

Consider the integral representation (see [9, p. 105])

$$
{ }_{1} F_{1}(a ; b ; z)=\frac{\Gamma(b) \Gamma(1+a-b)}{2 \pi i \Gamma(a)} \int_{0}^{\left(1^{+}\right)} e^{z t} t^{a-1}(t-1)^{b-a-1} d t,
$$


where $\Re a>0$ and $b-a \notin \mathbb{N}$. The contour starts and terminates at $t=0$, and encircles the point $t=1$ in the anti-clockwise direction.

Again we write $a=\gamma b$, and we expand at $t=\gamma$, the saddle point of $t^{a}(t-1)^{b-a}$,

$$
e^{z t}=e^{\gamma z} \sum_{n=0}^{\infty} \frac{z^{n}}{n !}(t-\gamma)^{n}
$$

and substitute this expansion into (37). This gives the expansion

$$
{ }_{1} F_{1}(a ; b ; z)=e^{\gamma z} \sum_{n=0}^{\infty} \frac{z^{n}}{n !} \Phi_{n},
$$

where

$$
\Phi_{n}=\frac{\Gamma(b) \Gamma(1+a-b)}{2 \pi i \Gamma(a)} \int_{0}^{\left(1^{+}\right)} t^{a-1}(t-1)^{b-a-1}(t-\gamma)^{n} d t
$$

We can write $\Phi_{n}$ in terms of the Gauss hypergeometric function:

$$
\Phi_{n}=(-\gamma)_{2}^{n} F_{1}(-n, a ; b ; 1 / \gamma)
$$

as follows from the representation of the Gauss function (see [9, p. 111]):

$$
{ }_{2} F_{1}(a, b ; c ; z)=\frac{\Gamma(c) \Gamma(1+b-c)}{2 \pi i \Gamma(b)} \int_{0}^{\left(1^{+}\right)} t^{b-1}(t-1)^{c-b-1}(1-t z)^{-a} d t
$$

where $\Re b>0$. From the recurrence relation satisfied by Gauss functions, we see that

$$
\Phi_{n}=\frac{n-1}{b+n-1}\left(\gamma(1-\gamma) \Phi_{n-2}+(1-2 \gamma) \Phi_{n-1}\right), \quad n=2,3, \ldots,
$$

with $\Phi_{0}=1, \Phi_{1}=0$. It is easily verified that all $\Phi_{n}, n \geq 2$, vanish when $\gamma=0$ or $\gamma=1$.

The expansion in (39) has an asymptotic character for large $b$, with $z$ and $\gamma$ bounded. This follows from the estimate

$$
\Phi_{n}=\mathcal{O}\left(b^{-\lfloor(n+1) / 2\rfloor}\right), \quad b \rightarrow \infty,
$$

which can be proved by using (43) and mathematical induction. On the other hand, the expansion is convergent. This can be easily seen by using the ratio test and considering that $\Phi_{n+1} / \Phi_{n}$ is bounded as $n \rightarrow+\infty$.

Taking into account the ranges of parameters for which the integral representations are valid, we conclude that (39) is an asymptotic (and convergent) expansion as $b \rightarrow$ 
$\infty$, with $\gamma, z$ bounded complex numbers, and $b-a \notin \mathbb{N}$. The restriction $b-a \notin \mathbb{N}$ can be eliminated by using the well-known integral representation

$$
{ }_{1} F_{1}(a ; b ; z)=\frac{\Gamma(b)}{\Gamma(a) \Gamma(b-a)} \int_{0}^{1} e^{z t} t^{a-1}(1-t)^{b-a-1} d t,
$$

valid for $\Re b>\Re a>0$ and proceeding similarly as before. The same expansion is obtained starting from this representation for which the restriction $b-a \notin \mathbb{N}$ is not present.

Observe that the relation (10) is preserved in expansion (39). We have

$$
\begin{aligned}
& { }_{1} \mathrm{~F}_{1}(\gamma b ; b ; z)=e^{z}{ }_{1} \mathrm{~F}_{1}((1-\gamma) b ; b ;-z)=e^{z} e^{(\gamma-1) z} \sum_{n=0}^{\infty} \frac{(-z)^{n}}{n !} \widetilde{\Phi}_{n} \\
& \widetilde{\Phi}_{n}=(1-\gamma)^{n}{ }_{2} \mathrm{~F}_{1}(-n,(1-\gamma) b ; b ; 1 /(1-\gamma))=(-1)^{n} \Phi_{n} .
\end{aligned}
$$

The expansion in (39) can be seen as convergent expansion in the complex domains of the parameters $b$ and $\gamma$, and the expansion constitutes an analytic function with respect to these parameters. The left-hand side of (39) is also an analytic function of these parameters, with the usual exception for $b=0,-1,-2, \ldots$, and analytic continuation can be used to extend the domain of convergence for all fixed complex $\gamma$ and complex $b \neq 0,-1,-2, \ldots$, with as extra property the asymptotic nature of the expansion as $b \rightarrow \infty$.

Acknowledgments The authors acknowledge financial support from Ministerio de Educación y Ciencia, project MTM2006-09050. NMT acknowledges the financial support from the Gobierno of Navarra, Res. 07/05/2008. The authors thank the two anonymous referees for useful comments and suggestions.

\section{References}

1. Abramowitz, M., Stegun, I.A: Handbook of Mathematical Functions with Formulas, Graphs, and Mathematical Tables, vol. 55. National Bureau of Standards Applied Mathematics Series, Washington, DC (1964)

2. Deaño, A., Segura, J.: Transitory minimal solutions of hypergeometric recursions and pseudoconvergence of associated continued fractions. Math. Comp. 76(258), 879-901 (2007)

3. Deaño, A., Segura, J., Temme, N.M.: Identifying minimal and dominant solutions for Kummer recursions. Math Comput (2008, in press)

4. Dunster, T.M.: Uniform asymptotic expansions for Whittaker's confluent hypergeometric functions. SIAM J. Math. Anal. 20(3), 744-760 (1989)

5. Gil, A., Segura, J., Temme, N.M.: Numerically satisfactory solutions of hypergeometric recursions. Math. Comp. 76(259), 1449-1468 (2007)

6. Gil, A., Segura, J., Temme, N.M.: Numerical Methods for Special functions. SIAM, Philadelphia (2007)

7. Olver, F.W.J.: Asymptotics and Special Functions. Academic Press, London (1974) (Reprinted by AK Peters)

8. Slater, L.J.: Confluent Hypergeometric Functions. Cambridge University Press, London (1960)

9. Temme, N.M.: Special Functions an Introduction to the Classical Functions of Mathematical Physics. Wiley, New York (1996) 